\title{
Nitrene Equivalents Mediated Metal-Free Ring Expansions of Alkylidenecyclopropanes and an Alkylidenecyclobutane
}

Yong Liang, Lei Jiao, Yuanyuan Wang, Yuanyuan Chen, Linge Ma, Jiaxi Xu, Shiwei Zhang, and Zhi-Xiang Yu*

Key Laboratory of Bioorganic Chemistry and Molecular Engineering of Ministry of Education, Beijing National Laboratory for Molecular Sciences, College of Chemistry, Peking University, Beijing 100871, China

yuzx@pku.edu.cn

\section{Supplemental Materials}

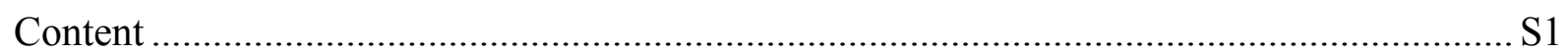

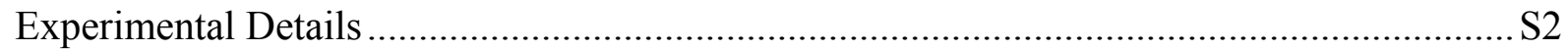

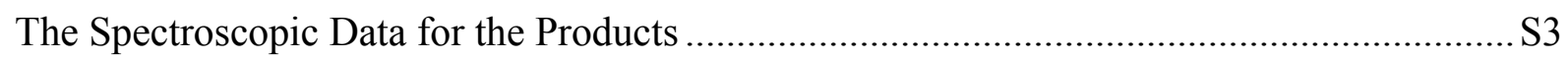

Copies of ${ }^{1} \mathrm{H}$ and ${ }^{13} \mathrm{C}$ NMR Spectra for the Products ..................................................... S8

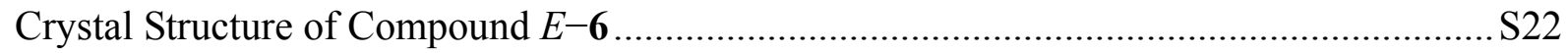




\section{Experimental Details}

General. $N$-aminophthalimide, ${ }^{1}$ ACPs $\left(\mathbf{1}, \mathbf{3}, \mathbf{5}, \mathbf{7}, \mathbf{9}, \mathbf{1 1}, \mathbf{1 3}\right.$, and 15), ${ }^{2}$ and ACB $21^{3}$ were prepared according to literature procedures. Dichloromethane and acetonitrile were refluxed with $\mathrm{CaH}_{2}$ and freshly distilled prior to use.

General Procedure for Metal-Free Ring Expansions of ACPs and ACBs. To a solution of ACP or ACB $(1 \mathrm{mmol})$ in $20 \mathrm{~mL} \mathrm{CH}_{2} \mathrm{Cl}_{2}$ was added $N$-aminophthalimide $(1.5 \mathrm{mmol})$ and (diacetoxyiodo)benzene $(1.5 \mathrm{mmol})$ successively. After stirred at room temperature for 2 hours, the reaction mixture was submitted to vacuum to remove the solvent. Column chromatography of the resulting crude mixture on silica gel afforded the corresponding products.

General Procedure for Copper-Catalyzed Ring Expansions of ACPs. To a solution of $\mathrm{ACP}(0.5 \mathrm{mmol})$ in $10 \mathrm{~mL} \mathrm{MeCN}$ was added $\mathrm{PhI}=\mathrm{NTs}(0.75 \mathrm{mmol})$ and $\mathrm{Cu}(\mathrm{acac})_{2}(0.05$ mmol) successively. After stirred at room temperature for 2 hours, the reaction mixture was submitted to vacuum to remove the solvent. Column chromatography of the resulting crude mixture on silica gel afforded the corresponding products.

(1) Christine, T. S.; Picard, J.; Yudin, A. K. J. Org. Chem. 2005, 70, 932-937.

(2) Utimoto, K.; Tamura, M.; Sisido, K. Tetrahedron 1973, 29, 1169-1171. ACP 7 was prepared by acetylization of (o-Aminophenyl)phenylmethylenecyclopropane, which was obtained according to the literature method. Spectroscopic data for unknown substrates: ACP 7: ${ }^{1} \mathrm{H}$ NMR $\left(300 \mathrm{MHz}, \mathrm{CDCl}_{3}\right): \delta 1.21$ $(\mathrm{t}, J=7.8 \mathrm{~Hz}, 2 \mathrm{H}), 1.65(\mathrm{t}, J=7.8 \mathrm{~Hz}, 2 \mathrm{H}), 1.78(\mathrm{~s}, 3 \mathrm{H}), 6.98(\mathrm{br}, 1 \mathrm{H}), 7.14-7.47(\mathrm{~m}, 8 \mathrm{H}), 8.22(\mathrm{~d}, J=8.4$ $\mathrm{Hz}, 1 \mathrm{H}) .{ }^{13} \mathrm{C}$ NMR $\left(75.5 \mathrm{MHz}, \mathrm{CDCl}_{3}\right): \delta 2.0,5.6,24.4,121.5,124.2,126.3,126.5,127.5,127.6,128.2$, 128.7, 130.4, 130.9, 135.6, 139.0, 168.0. ACP 11: ${ }^{1} \mathrm{H}$ NMR (300 MHz, $\left.\mathrm{CDCl}_{3}\right): \delta 1.15-1.37(\mathrm{~m}, 7 \mathrm{H}), 2.34$ (s, 3H), $2.57(\mathrm{t}, J=7.8 \mathrm{~Hz}, 2 \mathrm{H}), 2.98(\mathrm{t}, J=7.8 \mathrm{~Hz}, 2 \mathrm{H}), 4.12(\mathrm{q}, J=7.2 \mathrm{~Hz}, 2 \mathrm{H}), 7.16(\mathrm{~d}, J=8.1 \mathrm{~Hz}, 2 \mathrm{H})$, $7.49(\mathrm{~d}, J=8.1 \mathrm{~Hz}, 2 \mathrm{H}) .{ }^{13} \mathrm{C}$ NMR $\left(75.5 \mathrm{MHz}, \mathrm{CDCl}_{3}\right): \delta 1.4,4,3,14.2,21.0,29.2,33.2,60.3,120.3,125.5$, 125.7, 128.9, 136.3, 136.7, 173.5. ACP 15: ${ }^{1} \mathrm{H}$ NMR $\left(300 \mathrm{MHz}, \mathrm{CDCl}_{3}\right): \delta 1.04(\mathrm{t}, J=7.2 \mathrm{~Hz}, 2 \mathrm{H}), 1.43(\mathrm{t}$, $J=7.2 \mathrm{~Hz}, 2 \mathrm{H}), 1.86(\mathrm{~m}, 2 \mathrm{H}), 2.63(\mathrm{~m}, 2 \mathrm{H}), 2.83(\mathrm{t}, J=6.3 \mathrm{~Hz}, 2 \mathrm{H}), 7.09(\mathrm{~m}, 3 \mathrm{H}), 7.92(\mathrm{~d}, J=7.2 \mathrm{~Hz}, 1 \mathrm{H})$. ${ }^{13} \mathrm{C}$ NMR $\left(75.5 \mathrm{MHz}, \mathrm{CDCl}_{3}\right): \delta-0.4,5.3,23.2,30.5,31.5,116.7,125.1,125.7,126.2,128.9,133.3,135.1$, 136.7.

(3) Graham, S. H.; Williams, A. J. S. J. Chem. Soc. 1959, 4066-4073. 


\section{The Spectroscopic Data for the Products}

\section{N-phthalyl-2, 2-diphenylcyclobutylidene hydrazine (2)}<smiles>c1ccc(N=C2CCC2(c2ccccc2)c2ccccc2)cc1</smiles>

$E$-isomer (isolated yield: $21 \%)$ : white solid $\left(R_{f}=0.18, \mathrm{PE} / \mathrm{AcOEt}=5: 1\right)$, m.p. $176-178{ }^{\circ} \mathrm{C}$. ${ }^{1} \mathrm{H}$ NMR $\left(300 \mathrm{MHz}, \mathrm{CDCl}_{3}\right): \delta 2.86(\mathrm{t}, J=8.1 \mathrm{~Hz}, 2 \mathrm{H}), 3.04(\mathrm{t}, J=8.1 \mathrm{~Hz}, 2 \mathrm{H}), 7.21-7.38$ $(\mathrm{m}, 6 \mathrm{H}), 7.57-7.60(\mathrm{~m}, 4 \mathrm{H}), 7.72-7.77(\mathrm{~m}, 2 \mathrm{H}), 7.86-7.89(\mathrm{~m}, 2 \mathrm{H}) .{ }^{13} \mathrm{C}$ NMR $(75.5 \mathrm{MHz}$, $\left.\mathrm{CDCl}_{3}\right): \delta 29.5,33.2,64.6,123.5,126.8,127.0,128.5,131.0,134.2,143.5,163.7,183.8$. IR $v$ $\left(\mathrm{cm}^{-1}\right):$ 1719, 1730. MS (EI) m/z: $366\left(\mathrm{M}^{+}, 26\right), 220$ (47), 186 (65), 180 (76), 165 (100). Calcd for $\mathrm{C}_{24} \mathrm{H}_{18} \mathrm{~N}_{2} \mathrm{O}_{2}$ : C, 78.67; H, 4.95; N, 7.65. Found: C, 78.53; H, 4.96; N, 7.55. $Z$-isomer ${ }^{4}$ (isolated yield: 59\%): white solid $\left(R_{f}=0.11\right.$, PE/AcOEt $\left.=5: 1\right)$, m.p. $177-179{ }^{\circ} \mathrm{C}$. ${ }^{1} \mathrm{H}$ NMR (300 MHz, DMSO-d6): $\delta 2.71(\mathrm{t}, J=8.1 \mathrm{~Hz}, 2 \mathrm{H}), 3.14(\mathrm{t}, J=8.1 \mathrm{~Hz}, 2 \mathrm{H})$, 7.04-7.20 (m, 10H), 7.53-7.56 (m, 2H), 7.66-7.69 (m, 2H). ${ }^{13} \mathrm{C}$ NMR $(75.5 \mathrm{MHz}$, DMSO-d6): $\delta$ 28.7, 32.5, 69.6, 122.7, 126.8, 128.0, 128.2, 129.9, 134.1, 141.3, 162.9, 183.8 . IR $v\left(\mathrm{~cm}^{-1}\right): 1724$. MS (EI) m/z: $366\left(\mathrm{M}^{+}, 34\right), 220$ (37), 186 (72), 180 (95), 165 (69). Calcd for $\mathrm{C}_{24} \mathrm{H}_{18} \mathrm{~N}_{2} \mathrm{O}_{2}: 366.1368$. Found: 366.1359 .

\section{$N$-phthalyl-2-(4-methoxyphenyl)-2-phenylcyclobutylidene hydrazine (4)}<smiles>COc1ccc(C2(c3ccccc3)CCC2=Nc2ccccc2)cc1</smiles>

$E$-isomer (isolated yield: $43 \%)$ : colorless oil $\left(R_{f}=0.13, \mathrm{PE} / \mathrm{AcOEt}=5: 1\right) .{ }^{1} \mathrm{H} \mathrm{NMR}(300$ $\left.\mathrm{MHz}, \mathrm{CDCl}_{3}\right): \delta 2.81(\mathrm{t}, J=8.4 \mathrm{~Hz}, 2 \mathrm{H}), 3.03(\mathrm{t}, J=8.4 \mathrm{~Hz}, 2 \mathrm{H}), 3.77(\mathrm{~s}, 3 \mathrm{H}), 6.86-6.89(\mathrm{~m}$, 2H), 7.22-7.57 (m, 7H), 7.70-7.74 (m, 2H), 7.84-7.87 (m, 2H). ${ }^{13} \mathrm{C}$ NMR (75.5 MHz, $\left.\mathrm{CDCl}_{3}\right): \delta 29.6,33.1,55.2,64.0,113.8,123.4,126.7,127.0,128.2,128.4,130.9,134.1,135.7$, 143.8, 158.3, 163.7, 184.2. IR $v\left(\mathrm{~cm}^{-1}\right): 1720$. MS (EI) m/z: $396\left(\mathrm{M}^{+}, 13\right), 250$ (100), 210 (80). Calcd for $\mathrm{C}_{25} \mathrm{H}_{20} \mathrm{~N}_{2} \mathrm{O}_{3}: 396.1474$. Found: 396.1472.

$Z$-isomer (isolated yield: $55 \%)$ : white solid $\left(R_{f}=0.06\right.$, PE/AcOEt $\left.=5: 1\right)$, m.p. $153-155^{\circ} \mathrm{C}$. ${ }^{1} \mathrm{H}$ NMR (300 MHz, DMSO-d6): $\delta 2.58-2.78$ (m, 2H), $3.14(\mathrm{t}, J=8.4 \mathrm{~Hz}, 2 \mathrm{H}), 3.58(\mathrm{~s}, 3 \mathrm{H})$,

(4) This compound is not stable in $\mathrm{CDCl}_{3}$, and can be completely converted to $E$-isomer after 3 days. 
$6.63(\mathrm{~d}, J=9.0 \mathrm{~Hz}, 2 \mathrm{H}), 7.05-7.25(\mathrm{~m}, 7 \mathrm{H}), 7.56-7.60(\mathrm{~m}, 2 \mathrm{H}), 7.69-7.72(\mathrm{~m}, 2 \mathrm{H}) .{ }^{13} \mathrm{C}$ NMR (75.5 MHz, DMSO-d6): $\delta$ 28.7, 32.5, 54.9, 69.1, 113.3, 122.6, 126.7, 128.0, 128.2, 129.3, 130.0, 132.8, 134.0, 141.8, 157.9, 162.8, 184.1. IR $v\left(\mathrm{~cm}^{-1}\right): 1724$. MS (EI) m/z: 396 $\left(\mathrm{M}^{+}, 14\right), 250$ (100), 210 (70). Calcd for $\mathrm{C}_{25} \mathrm{H}_{20} \mathrm{~N}_{2} \mathrm{O}_{3}$ : C, 75.74; H, 5.08; N, 7.07. Found: C, 75.75; H, 5.07; N, 7.04.

\section{$\mathrm{N}$-phthalyl-2-(3-chlorophenyl)-2-phenylcyclobutylidene hydrazine (6)}

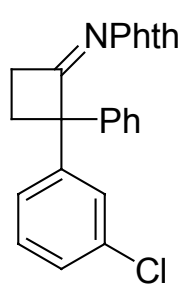

$E$-isomer (isolated yield: $36 \%$ ): white solid $\left(R_{f}=0.17\right.$, PE/AcOEt $\left.=5: 1\right)$, m.p. $185-187{ }^{\circ} \mathrm{C}$. ${ }^{1} \mathrm{H}$ NMR $\left(300 \mathrm{MHz} \mathrm{CDCl}_{3}\right): \delta 2.80-2.91(\mathrm{~m}, 2 \mathrm{H}), 3.05(\mathrm{t}, J=8.4 \mathrm{~Hz}, 2 \mathrm{H}), 7.20-7.59(\mathrm{~m}$, 9H), 7.72-7.75 (m, 2H), 7.86-7.89 (m, 2H). $\left.{ }^{13} \mathrm{C} \mathrm{NMR} \mathrm{(75.5} \mathrm{MHz,} \mathrm{CDCl}_{3}\right): \delta 29.4,33.2,64.2$, $123.5,125.3,126.9,127.0,127.08,127.15$ 128.7, 129.8, 130.9, 134.2, 134.3, 142.8, 145.6, 163.6, 182.7. IR $v\left(\mathrm{~cm}^{-1}\right):$ 1718. MS (EI) m/z: $400\left(\mathrm{M}^{+}, 15\right), 254(21), 214$ (17), 186(100). Calcd for $\mathrm{C}_{24} \mathrm{H}_{17} \mathrm{ClN}_{2} \mathrm{O}_{2}$ : C, 71.91; H, 4.27; N, 6.99. Found: C, 71.90; H, 4.26; N, 6.92.

$Z$-isomer (isolated yield: $52 \%)$ : white solid $\left(R_{f}=0.10\right.$, PE/AcOEt $\left.=5: 1\right)$, m.p. $154-156{ }^{\circ} \mathrm{C}$. ${ }^{1} \mathrm{H}$ NMR (300 MHz, DMSO-d6): $\delta 2.66(\mathrm{~m}, 1 \mathrm{H}), 2.85(\mathrm{~m}, 1 \mathrm{H}), 3.19(\mathrm{t}, J=8.1 \mathrm{~Hz}, 2 \mathrm{H})$, 7.13-7.27 (m, 9H), 7.59-7.62 (m, 2H), 7.71-7.74 (m, 2H). ${ }^{13} \mathrm{C}$ NMR (75.5 MHz, DMSO-d6): $\delta 28.7,32.5,69.0,122.8,126.8,127.1,128.1,128.3,129.8,129.9,132.9,134.3,141.1,143.2$, 162.9, 183.2. IR $v\left(\mathrm{~cm}^{-1}\right):$ 1717. MS (EI) m/z: $400\left(\mathrm{M}^{+}, 12\right), 254(22), 214$ (18), 186(100). Calcd for $\mathrm{C}_{24} \mathrm{H}_{17} \mathrm{ClN}_{2} \mathrm{O}_{2}$ : C, 71.91; H, 4.27; N, 6.99. Found: C, 71.98; H, 4.24; N, 6.97.

\section{N-phthalyl-2-(2-acetamidophenyl)-2-phenylcyclobutylidene hydrazine (8)}

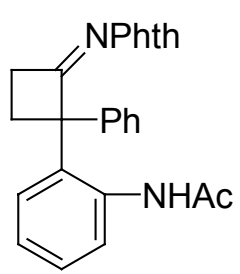

White solid $\left(R_{f}=0.09, \mathrm{PE} / \mathrm{AcOEt}=2: 1\right)$, m.p. $205-207{ }^{\circ} \mathrm{C} .{ }^{1} \mathrm{H} \mathrm{NMR}\left(300 \mathrm{MHz}, \mathrm{CDCl}_{3}\right): \delta$ $1.87(\mathrm{~s}, 3 \mathrm{H}), 2.50(\mathrm{~m}, 1 \mathrm{H}), 3.03-3.27(\mathrm{~m}, 3 \mathrm{H}), 7.20-7.38(\mathrm{~m}, 7 \mathrm{H}), 7.60(\mathrm{~d}, J=7.8 \mathrm{~Hz}, 1 \mathrm{H})$, $7.73(\mathrm{~d}, J=7.8 \mathrm{~Hz}, 1 \mathrm{H}), 7.77-7.80(\mathrm{~m}, 2 \mathrm{H}), 7.89-7.92(\mathrm{~m}, 2 \mathrm{H}), 9.62(\mathrm{~s}, 1 \mathrm{H}) .{ }^{13} \mathrm{C} \mathrm{NMR}(75.5$ $\left.\mathrm{MHz}, \mathrm{CDCl}_{3}\right): \delta 23.8,28.7,32.7,64.0,123.8,124.8,126.0,126.9,127.0,127.1128 .3,128.6$, 130.7, 134.1, 134.5, 136.3, 143.0, 163.4, 168.3, 184.5. IR $v\left(\mathrm{~cm}^{-1}\right): 1691,1715 . \mathrm{MS}(\mathrm{EI}) \mathrm{m} / \mathrm{z}$ : $423\left(\mathrm{M}^{+}, 17\right), 380$ (38), 218 (56). Calcd for $\mathrm{C}_{26} \mathrm{H}_{21} \mathrm{~N}_{3} \mathrm{O}_{3}$ : C, 73.74; H, 5.00; N, 9.92. Found: C, 
73.37; H, 5.01; N, 9.87.

N-phthalyl-2-(4-bromophenyl)-2-methylcyclobutylidene hydrazine (10)<smiles>CC1(c2ccc(Br)cc2)CCC1=Nc1ccccc1</smiles>

White solid $\left(R_{f}=0.18, \mathrm{PE} / \mathrm{AcOEt}=5: 1\right)$, m.p. $148-150{ }^{\circ} \mathrm{C} .{ }^{1} \mathrm{H}$ NMR $\left(300 \mathrm{MHz}, \mathrm{CDCl}_{3}\right): \delta$ $1.73(\mathrm{~s}, 3 \mathrm{H}), 2.24(\mathrm{~m}, 1 \mathrm{H}), 2.45(\mathrm{~m}, 1 \mathrm{H}), 3.00(\mathrm{t}, J=8.4 \mathrm{~Hz}, 2 \mathrm{H}), 7.44-7.52(\mathrm{~m}, 4 \mathrm{H})$, 7.74-7.77 (m, 2H), 7.87-7.90 (m, 2H). ${ }^{13} \mathrm{C} \mathrm{NMR}\left(75.5 \mathrm{MHz}, \mathrm{CDCl}_{3}\right): \delta 28.1,29.6,33.1,56.5$, 120.6, 123.5, 127.8, 130.9, 131.6, 134.2, 143.4, 163.8, 184.9. IR $v\left(\mathrm{~cm}^{-1}\right): 1722$. MS (EI) m/z: $382\left(\mathrm{M}^{+}, 6\right), 236$ (12), 186 (100). Calcd for $\mathrm{C}_{19} \mathrm{H}_{15} \mathrm{BrN}_{2} \mathrm{O}_{2}$ : C, 59.55; H, 3.95; N, 7.31. Found: C, 59.61; H, 3.97; N, 7.29.

$N$-phthalyl-2-(2-(ethoxycarbonyl)ethyl)-2-(4-methylphenyl)cyclobutylidene hydrazine (12)<smiles>CCOC(=O)CCC1(c2ccc(C)cc2)CCC1=Nc1ccccc1</smiles>

Colorless oil $\left(R_{f}=0.12, \mathrm{PE} / \mathrm{AcOEt}=5: 1\right) .{ }^{1} \mathrm{H} \mathrm{NMR}\left(300 \mathrm{MHz}, \mathrm{CDCl}_{3}\right): \delta 1.20(\mathrm{t}, J=7.2 \mathrm{~Hz}$, $3 \mathrm{H}), 2.34(\mathrm{~s}, 3 \mathrm{H}), 2.19-2.59(\mathrm{~m}, 6 \mathrm{H}), 2.94(\mathrm{t}, J=8.4 \mathrm{~Hz}, 2 \mathrm{H}), 4.06$ (q, $J=7.2 \mathrm{~Hz}, 2 \mathrm{H}), 7.19$ $(\mathrm{d}, J=8.1 \mathrm{~Hz}, 2 \mathrm{H}), 7.45(\mathrm{~d}, J=8.1 \mathrm{~Hz}, 2 \mathrm{H}), 7.73-7.76(\mathrm{~m}, 2 \mathrm{H}), 7.85-7.88(\mathrm{~m}, 2 \mathrm{H}) .{ }^{13} \mathrm{C}$ NMR (75.5 MHz, $\mathrm{CDCl}_{3}$ ): $\delta 14.1,20.9,27.7,30.0,32.5,35.7,59.5,60.2,123.4,126.5,129.1$, 130.9, 134.1, 136.5, 138.4, 163.6, 173.2, 184.4. IR $v\left(\mathrm{~cm}^{-1}\right): 1719$. MS (EI) m/z: $404\left(\mathrm{M}^{+}, 6\right)$, 317 (24), 186 (65), 145 (100). Calcd for $\mathrm{C}_{24} \mathrm{H}_{24} \mathrm{~N}_{2} \mathrm{O}_{4}$ : 404.1736. Found: 404. 1730.

$N$-phthalylcyclobutylidene hydrazine derivative 16

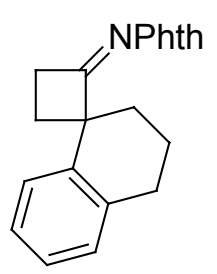

White solid $\left(R_{f}=0.18, \mathrm{PE} / \mathrm{AcOEt}=5: 1\right)$, m.p. $186-188^{\circ} \mathrm{C} .{ }^{1} \mathrm{H}$ NMR $\left(300 \mathrm{MHz}, \mathrm{CDCl}_{3}\right): \delta$ 
$1.87(\mathrm{~m}, 1 \mathrm{H}), 2.04(\mathrm{~m}, 1 \mathrm{H}), 2.21(\mathrm{~m}, 2 \mathrm{H}), 2.33(\mathrm{~m}, 2 \mathrm{H}), 2.84(\mathrm{~m}, 2 \mathrm{H}), 3.13(\mathrm{t}, J=8.4 \mathrm{~Hz}, 2 \mathrm{H})$, 7.07-7.27 (m, 3H), $7.50(\mathrm{~d}, J=7.8 \mathrm{~Hz}, 1 \mathrm{H}), 7.72-7.76(\mathrm{~m}, 2 \mathrm{H}), 7.85-7.88(\mathrm{~m}, 2 \mathrm{H}) .{ }^{13} \mathrm{C}$ NMR (75.5 MHz, $\left.\mathrm{CDCl}_{3}\right): \delta 19.6,29.5,32.0,33.1,34.4,56.1,123.4,126.5,126.8,127.5$, 129.0, 131.0, 134.1, 136.3, 138.4, 163.7, 188.5. IR $v\left(\mathrm{~cm}^{-1}\right): 1715 . \mathrm{MS}(\mathrm{EI}) \mathrm{m} / \mathrm{z}: 330\left(\mathrm{M}^{+}, 20\right)$, 186 (38), 144 (81), 129 (100). Calcd for $\mathrm{C}_{21} \mathrm{H}_{18} \mathrm{~N}_{2} \mathrm{O}_{2}$ : C, 76.34; H, 5.49; N, 8.48. Found: C, $76.25 ; \mathrm{H}, 5.45 ; \mathrm{N}, 8.45$.

\section{$N$-aminophthalimide derivative 17}

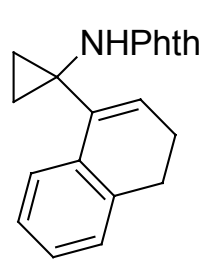

White solid $\left(R_{f}=0.22, \mathrm{PE} / \mathrm{AcOEt}=5: 1\right)$, m.p. $147-149{ }^{\circ} \mathrm{C} .{ }^{1} \mathrm{H} \mathrm{NMR}\left(300 \mathrm{MHz}, \mathrm{CDCl}_{3}\right): \delta$ $0.90(\mathrm{dd}, J=4.5,6.6 \mathrm{~Hz}, 2 \mathrm{H}), 1.49(\mathrm{dd}, J=4.5,6.6 \mathrm{~Hz}, 2 \mathrm{H}), 2.13(\mathrm{~m}, 3 \mathrm{H}), 2.68(\mathrm{t}, J=8.1 \mathrm{~Hz}$, 2H), $5.93(\mathrm{t}, J=4.8 \mathrm{~Hz}, 1 \mathrm{H}), 7.16-7.22(\mathrm{~m}, 3 \mathrm{H}), 7.68-7.78(\mathrm{~m}, 5 \mathrm{H}) .{ }^{13} \mathrm{C} \mathrm{NMR}(75.5 \mathrm{MHz}$, $\left.\mathrm{CDCl}_{3}\right): \delta 12.8,22.9,27.6,44.0,122.5,123.1,126.2,126.9,127.9,128.9,130.2,133.9,135.6$, 137.0, 166.1. IR $v\left(\mathrm{~cm}^{-1}\right): 1721$. MS (EI) m/z: $330\left(\mathrm{M}^{+}, 36\right), 169$ (79), 128 (100). Calcd for $\mathrm{C}_{21} \mathrm{H}_{18} \mathrm{~N}_{2} \mathrm{O}_{2}$ : C, 76.34; H, 5.49; N, 8.48. Found: C, 76.30; H, 5.44; N, 8.45.

\section{N-phthalyl-2, 2-diphenylcyclopentylidene hydrazine (22)}

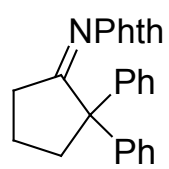

White solid $\left(R_{f}=0.20, \mathrm{PE} / \mathrm{AcOEt}=5: 1\right)$, m.p. $160-161{ }^{\circ} \mathrm{C} .{ }^{1} \mathrm{H}$ NMR $\left(300 \mathrm{MHz}, \mathrm{CDCl}_{3}\right): \delta$ $1.78(\mathrm{~m}, 2 \mathrm{H}), 2.66(\mathrm{t}, J=7.5 \mathrm{~Hz}, 2 \mathrm{H}), 2.73$ (t, $J=6.6 \mathrm{~Hz}, 2 \mathrm{H}), 7.24-7.39(\mathrm{~m}, 6 \mathrm{H}), 7.46-7.49$ (m, 4H), 7.69-7.72 (m, 2H), 7.82-7.85 (m, 2H). $\left.{ }^{13} \mathrm{C} \mathrm{NMR} \mathrm{(75.5} \mathrm{MHz,} \mathrm{CDCl}_{3}\right): \delta 20.2,31.9$, 40.7, 61.8, 123.3, 126.8, 128.2, 128.6, 131.2, 134.0, 142.5, 163.6, 194.1. IR $v\left(\mathrm{~cm}^{-1}\right): 1715$. MS (EI) m/z: $380\left(M^{+}, 20\right), 352$ (22), 234 (28), 115 (100). Calcd for $\mathrm{C}_{25} \mathrm{H}_{20} \mathrm{~N}_{2} \mathrm{O}_{2}$ : C, 78.93; H, 5.30; N, 7.36. Found: C, 79.05; H, 5.33; N, 7.34.

\section{$N$-tosyl-2, 2-diphenylcyclobutanimine (23)}<smiles>[N+]=C1CCC1(c1ccccc1)c1ccccc1</smiles>

White solid $\left(R_{f}=0.30, \mathrm{PE} / \mathrm{AcOEt}=5: 1\right)$, m.p. $114-116{ }^{\circ} \mathrm{C} .{ }^{1} \mathrm{H} \mathrm{NMR}\left(300 \mathrm{MHz}, \mathrm{CDCl}_{3}\right): \delta$ 
2.44 (s, 3H), 2.95 (t, $J=8.6 \mathrm{~Hz}, 2 \mathrm{H}), 3.52(\mathrm{t}, J=8.6 \mathrm{~Hz}, 2 \mathrm{H}), 7.19-7.38(\mathrm{~m}, 12 \mathrm{H}), 7.88(\mathrm{~d}, J$ $=8.4 \mathrm{~Hz}, 2 \mathrm{H}) .{ }^{13} \mathrm{C} \mathrm{NMR}\left(75.5 \mathrm{MHz}, \mathrm{CDCl}_{3}\right): \delta 21.6,29.9,36.5,67.1,126.5,127.0,127.4$, 128.6, 129.6, 136.9, 142.5, 144.2, 195.5. IR $v\left(\mathrm{~cm}^{-1}\right): 1659$. MS (EI) m/z: $375\left(\mathrm{M}^{+}, 1\right), 220$ (100), 91 (33). Calcd for $\mathrm{C}_{23} \mathrm{H}_{21} \mathrm{NO}_{2} \mathrm{~S}$ : C, 73.57; H, 5.64; N, 3.73. Found: C, 73.52; H, 5.65; $\mathrm{N}, 3.60$.

N-tosyl-2-(4-methoxyphenyl)-2-phenylcyclobutanimine (24)<smiles></smiles>

Colorless oil $\left(R_{f}=0.22, \mathrm{PE} / \mathrm{AcOEt}=5: 1\right) .{ }^{1} \mathrm{H} \mathrm{NMR}\left(300 \mathrm{MHz}, \mathrm{CDCl}_{3}\right): \delta 2.44(\mathrm{~s}, 3 \mathrm{H}), 2.90(\mathrm{t}$, $J=8.4 \mathrm{~Hz}, 2 \mathrm{H}), 3.51(\mathrm{t}, J=8.4 \mathrm{~Hz}, 2 \mathrm{H}), 3.74(\mathrm{~s}, 3 \mathrm{H}), 6.78-6.81(\mathrm{~m}, 2 \mathrm{H}), 7.18-7.35(\mathrm{~m}, 9 \mathrm{H})$, $7.87(\mathrm{~d}, J=8.4 \mathrm{~Hz}, 2 \mathrm{H}) .{ }^{13} \mathrm{C} \mathrm{NMR}\left(75.5 \mathrm{MHz}, \mathrm{CDCl}_{3}\right): \delta 21.6,30.0,36.4,55.2,66.6,113.9$, 126.5, 126.9, 127.4, 127.7, 128.6, 129.6, 134.6, 136.9, 142.9, 144.2, 158.5, 195.8. IR $v\left(\mathrm{~cm}^{-1}\right)$ : 1658. MS (EI) m/z: $405\left(\mathrm{M}^{+}, 1\right), 250$ (100), 210 (18). Calcd for $\mathrm{C}_{24} \mathrm{H}_{23} \mathrm{NO}_{3} \mathrm{~S}: 405.1399$. Found: 405.1394. 
Copies of ${ }^{1} \mathrm{H}$ and ${ }^{13} \mathrm{C}$ NMR Spectra for the Products
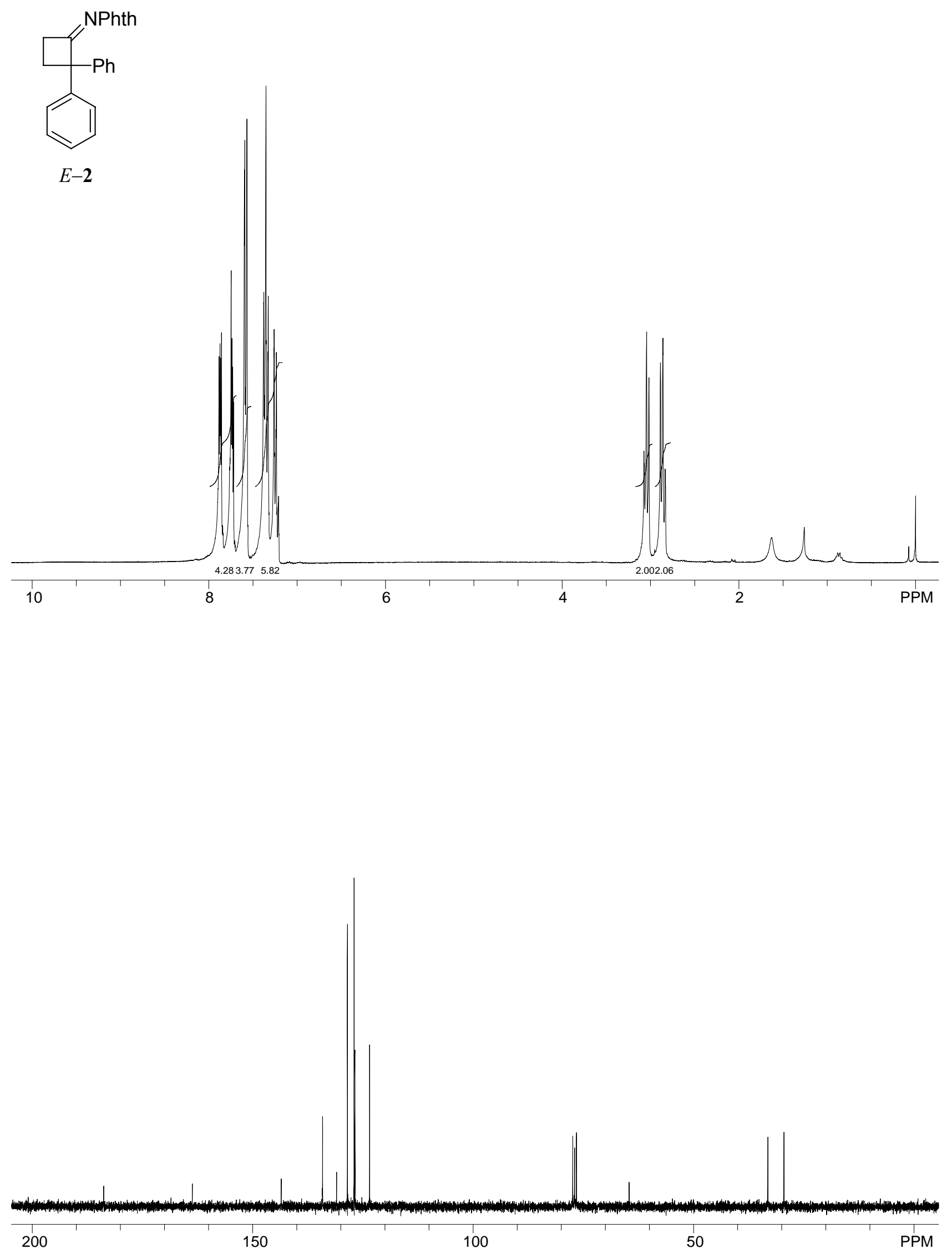

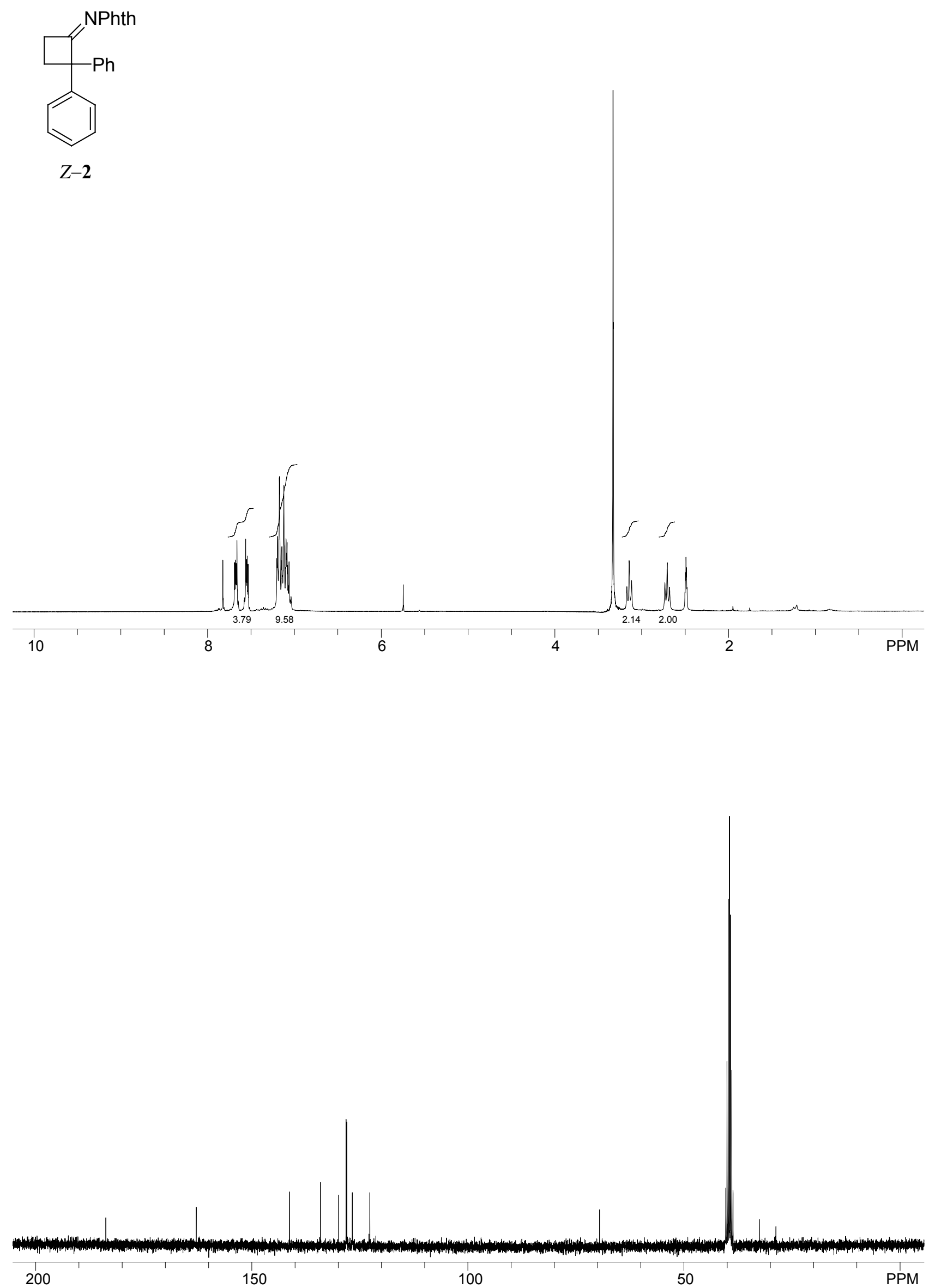

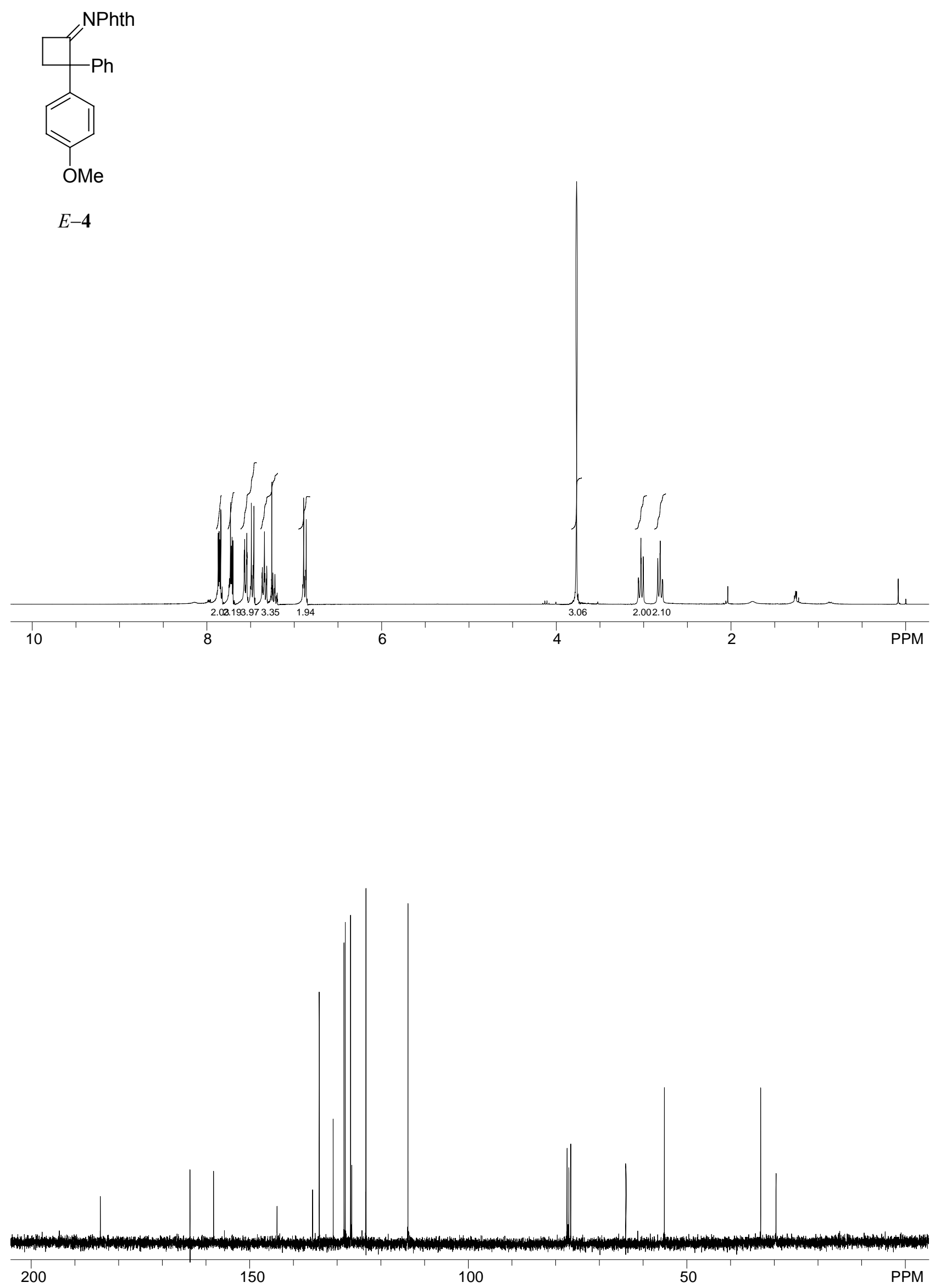

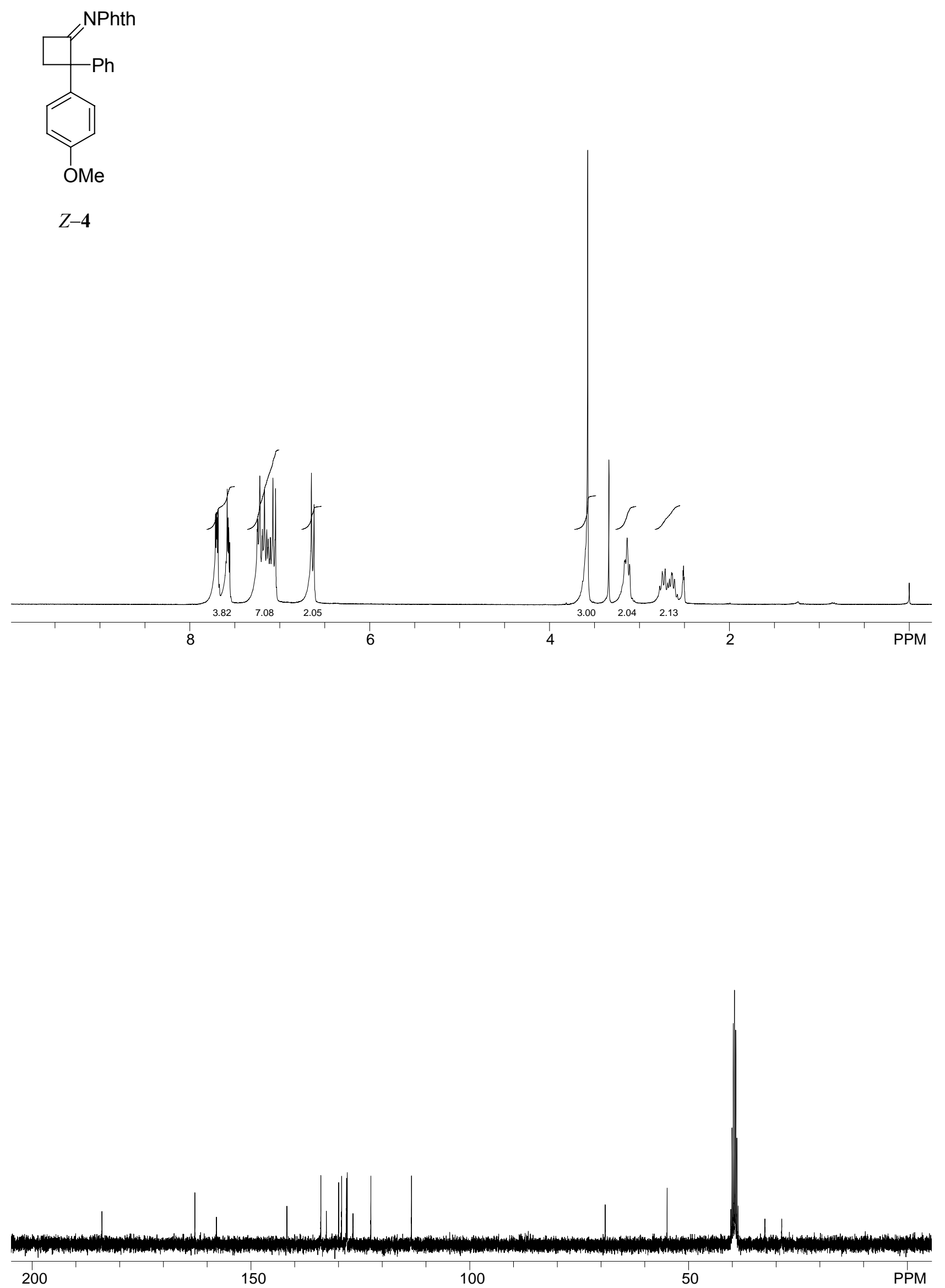


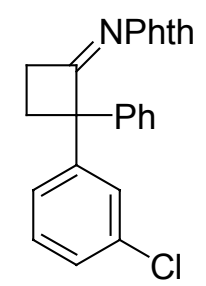

E-6
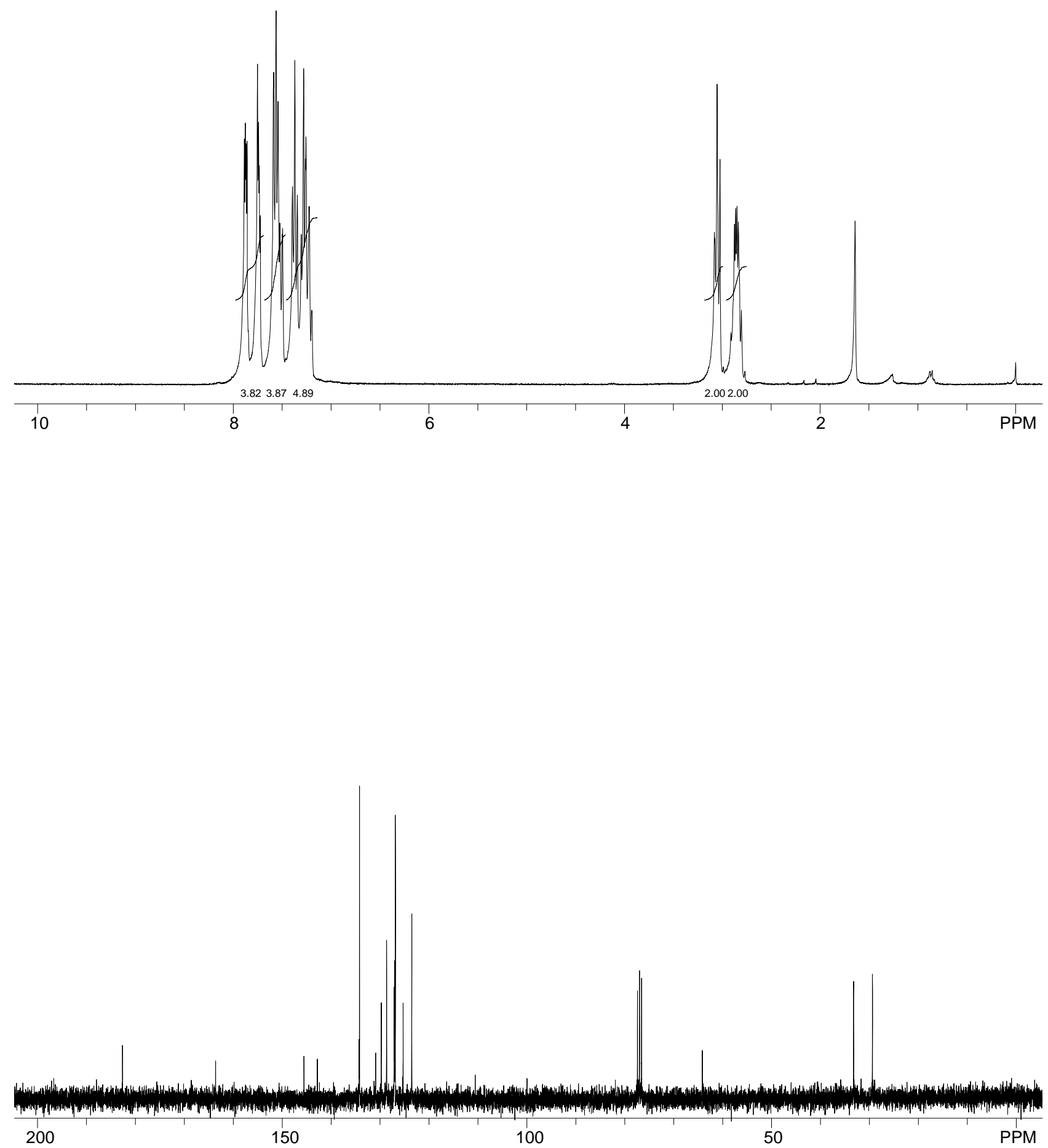

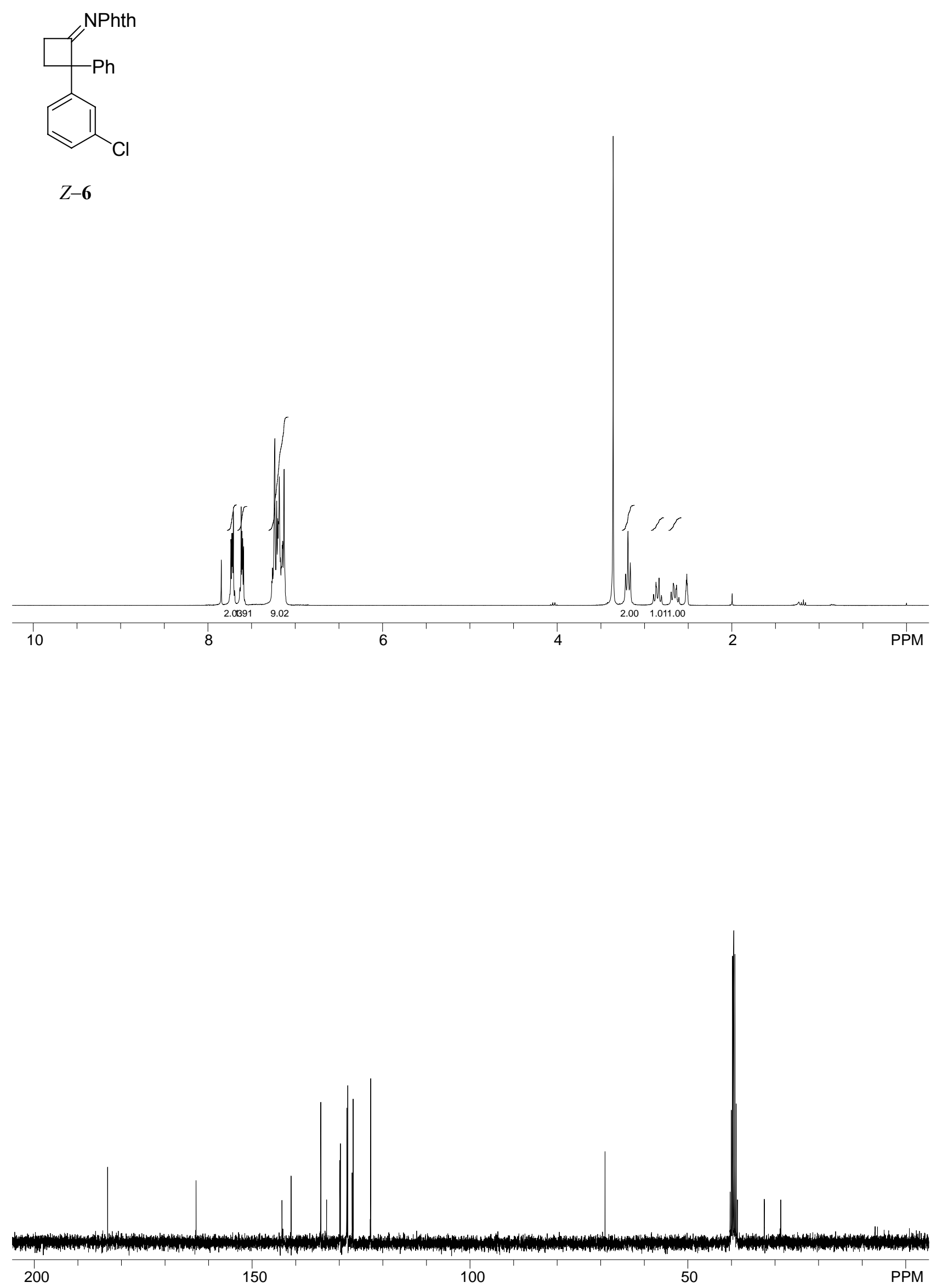
<smiles>CC(C)Nc1ccccc1C1(c2ccccc2)CCC1=Nc1ccccc1</smiles>

8
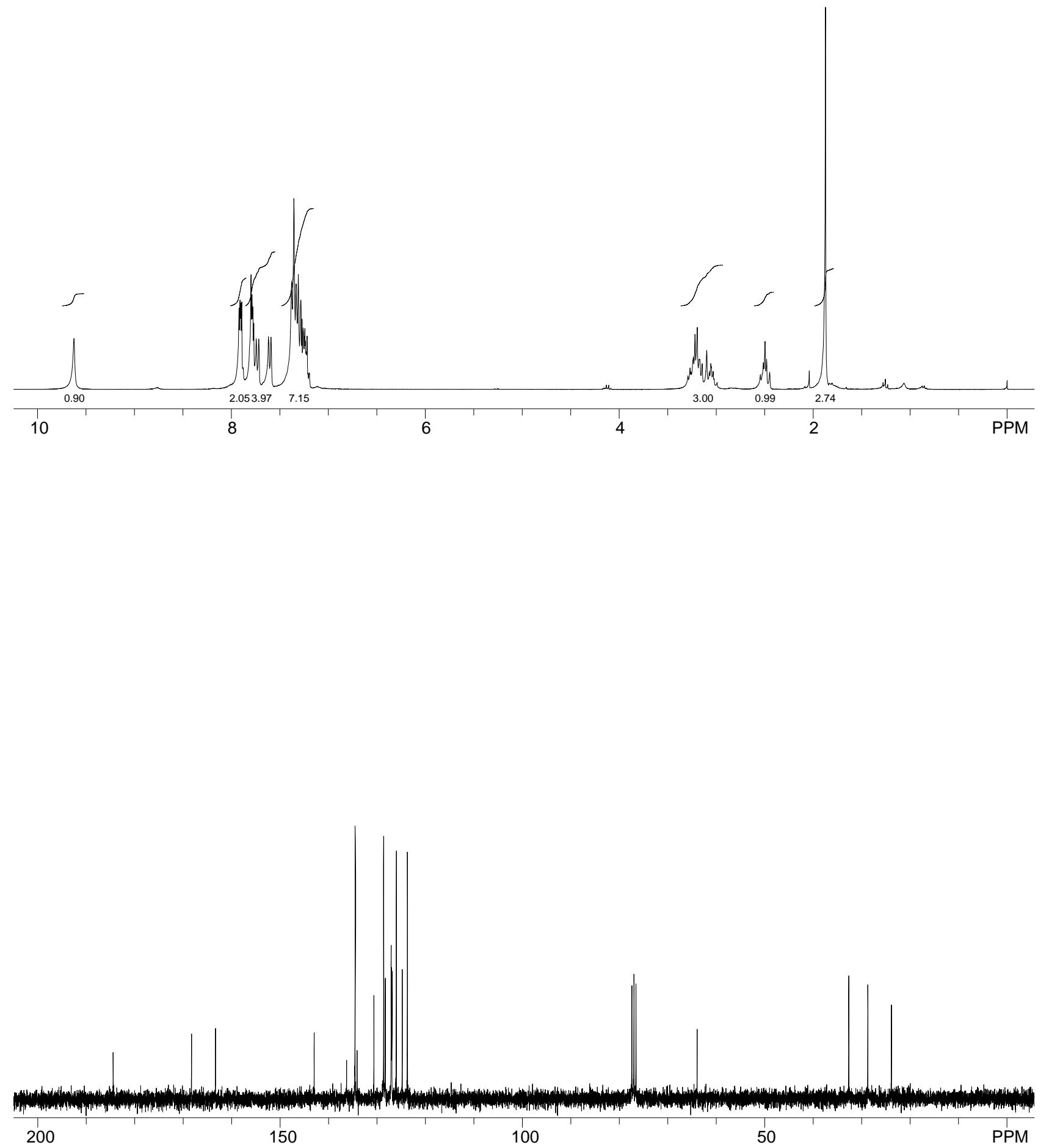

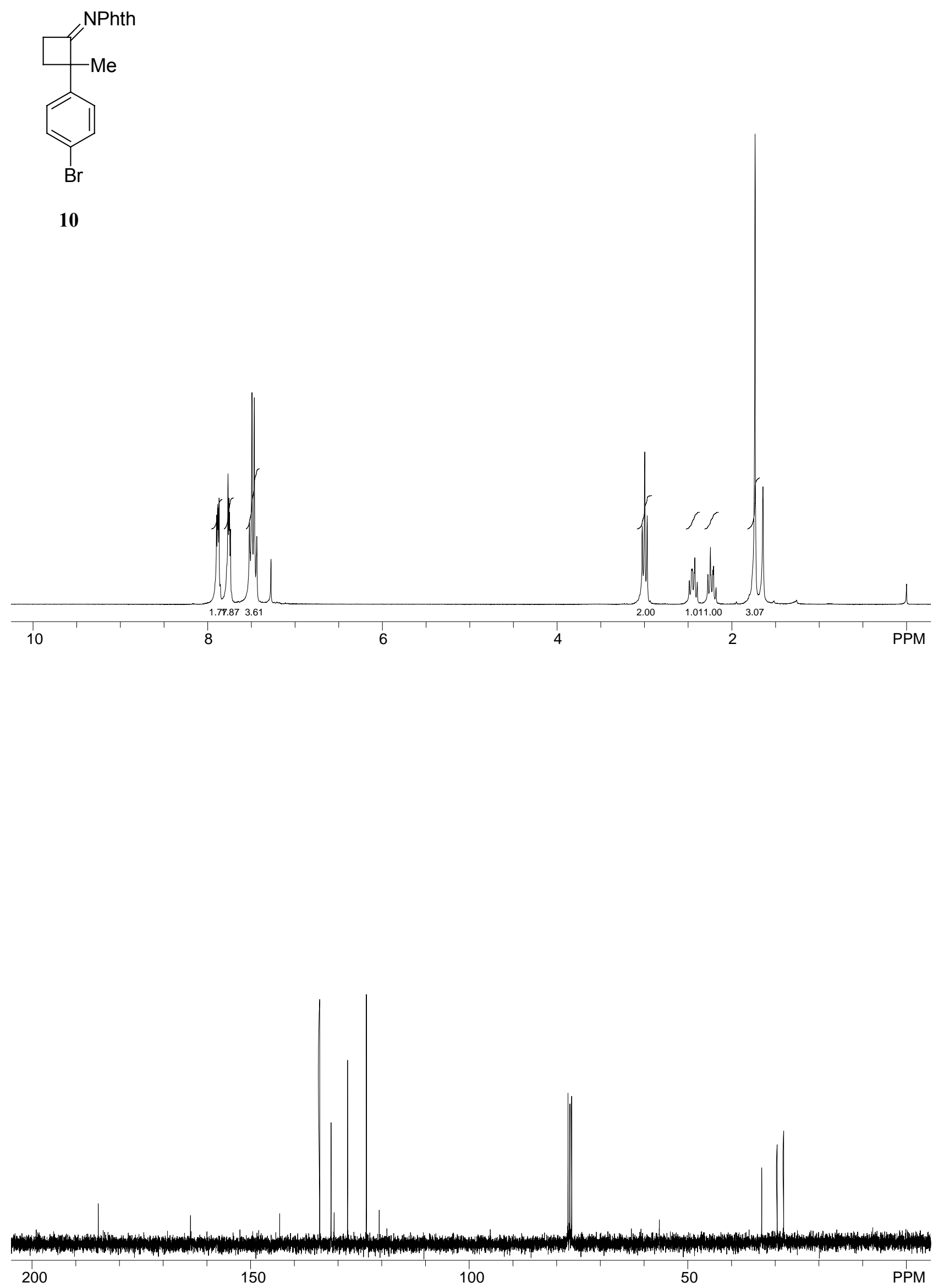


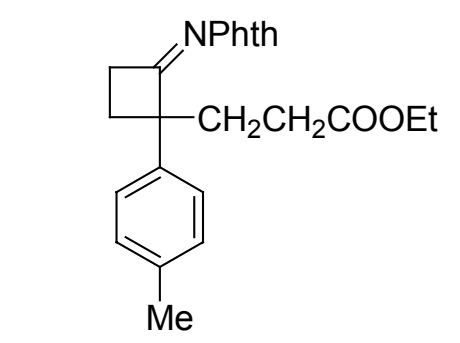

12
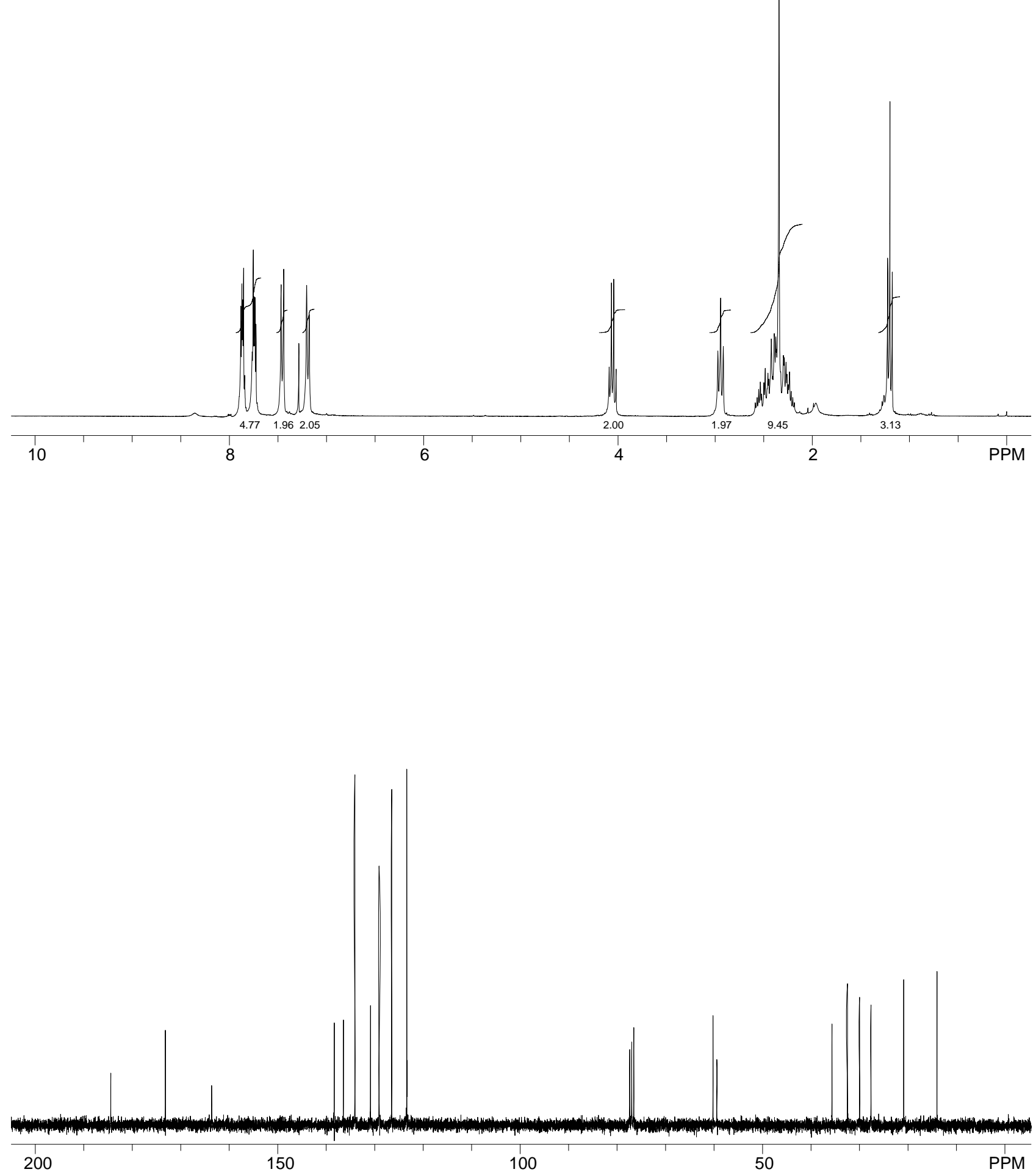


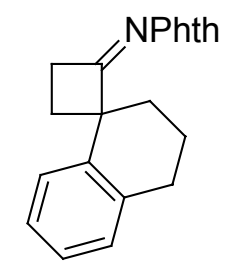

16
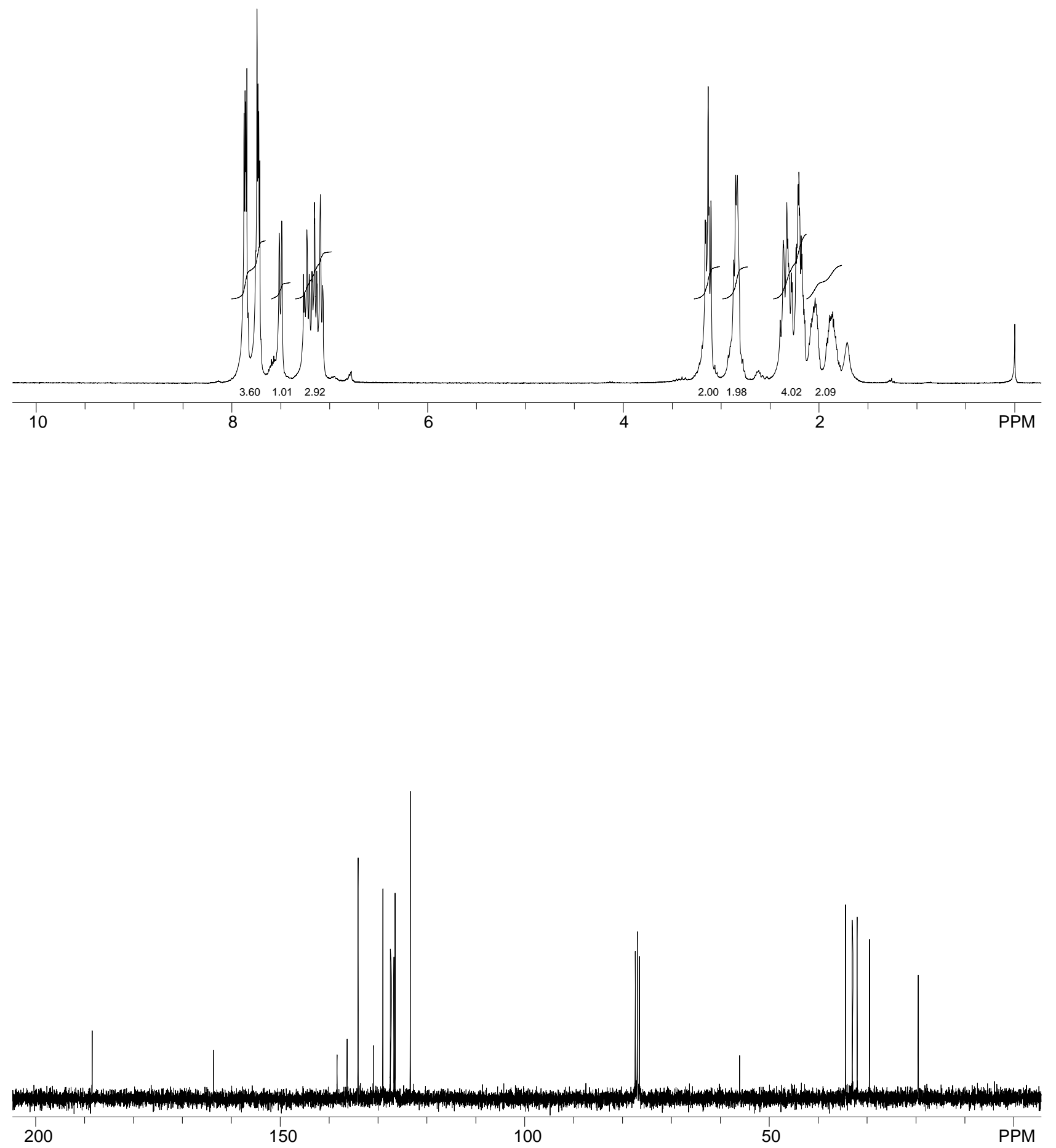


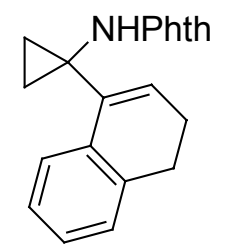

17
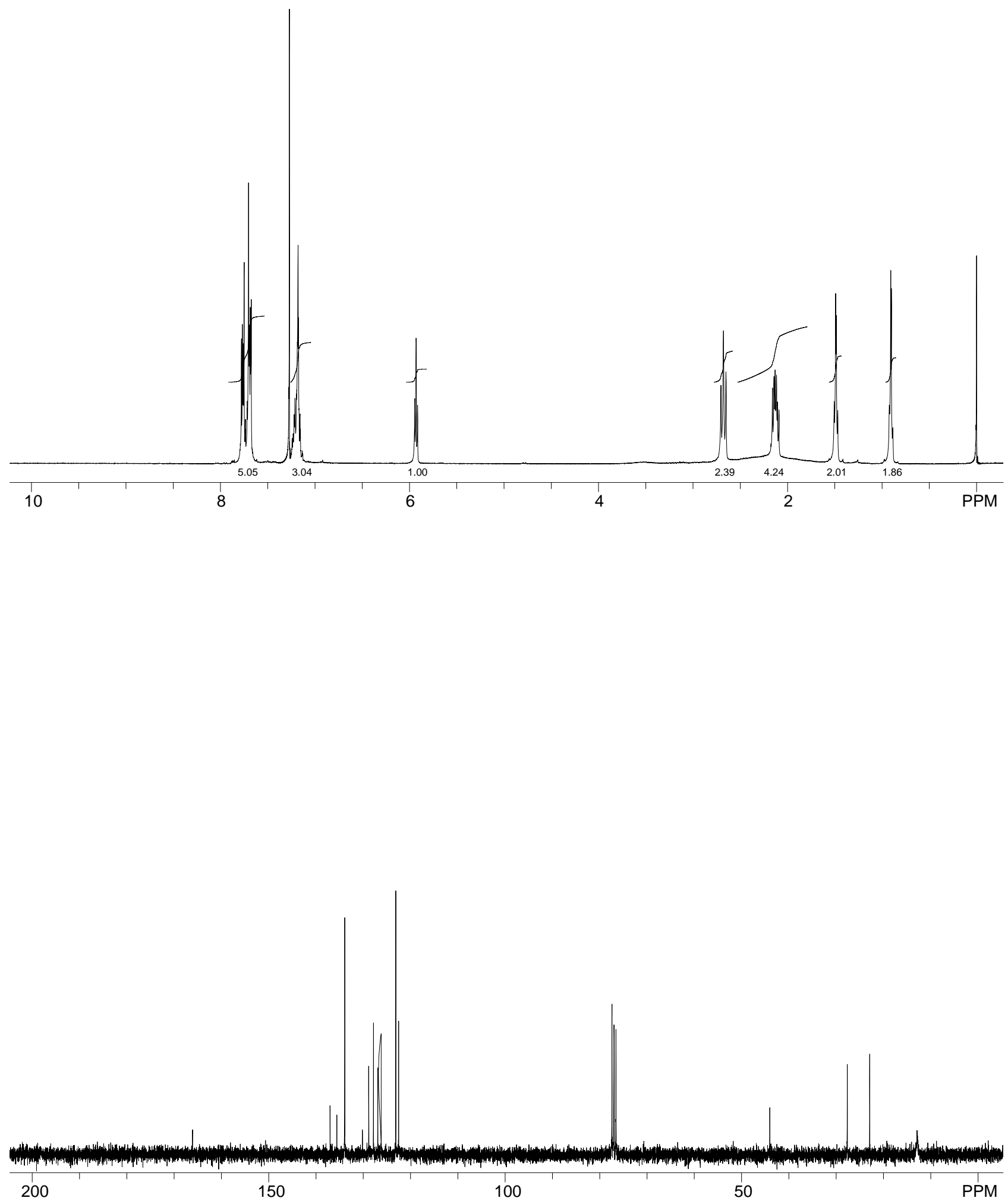
${ }_{X_{P h}^{N P h t h}}^{P h}$

22
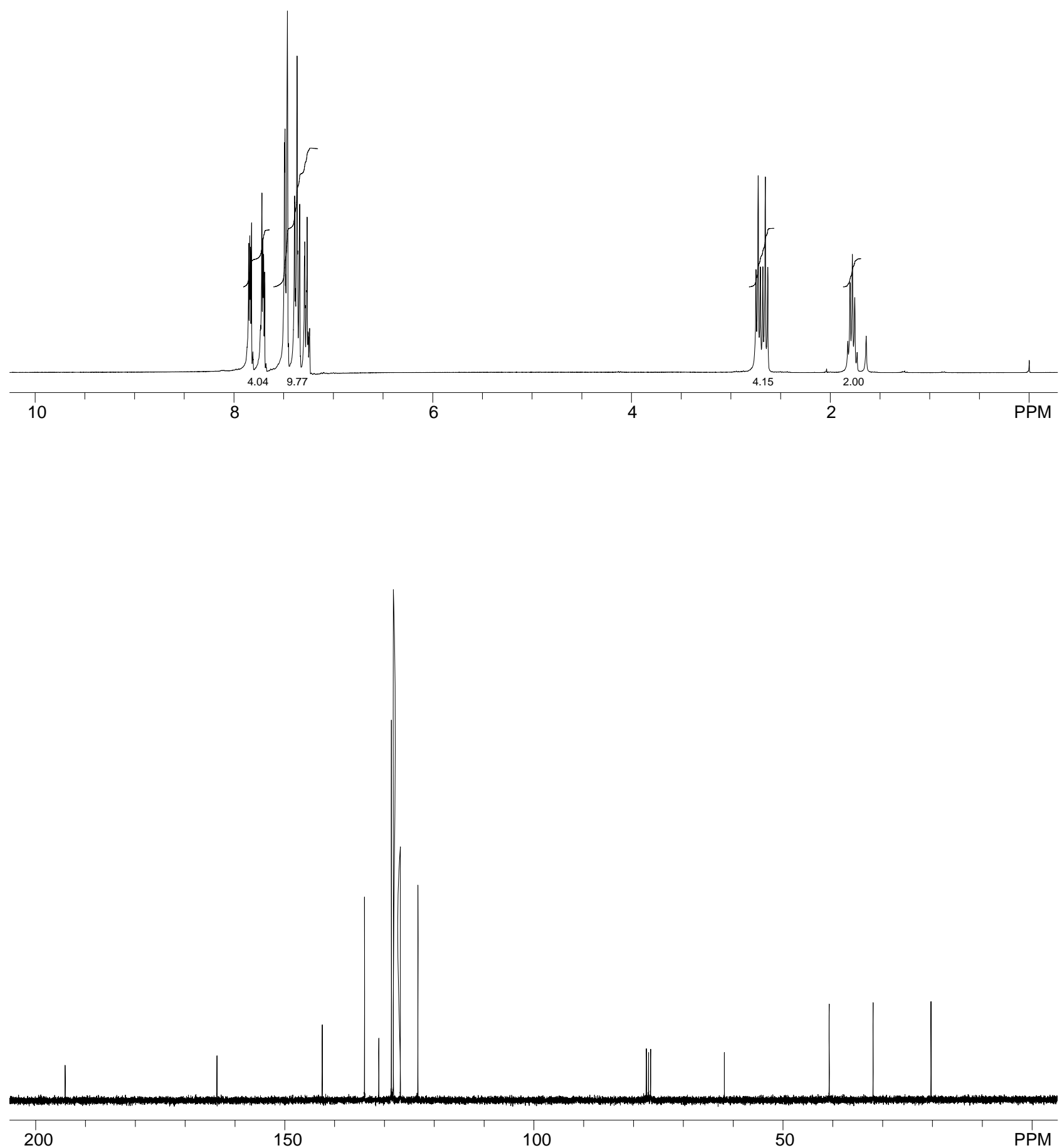
<smiles>[N+]=C1CCC1(c1ccccc1)c1ccccc1</smiles>

23
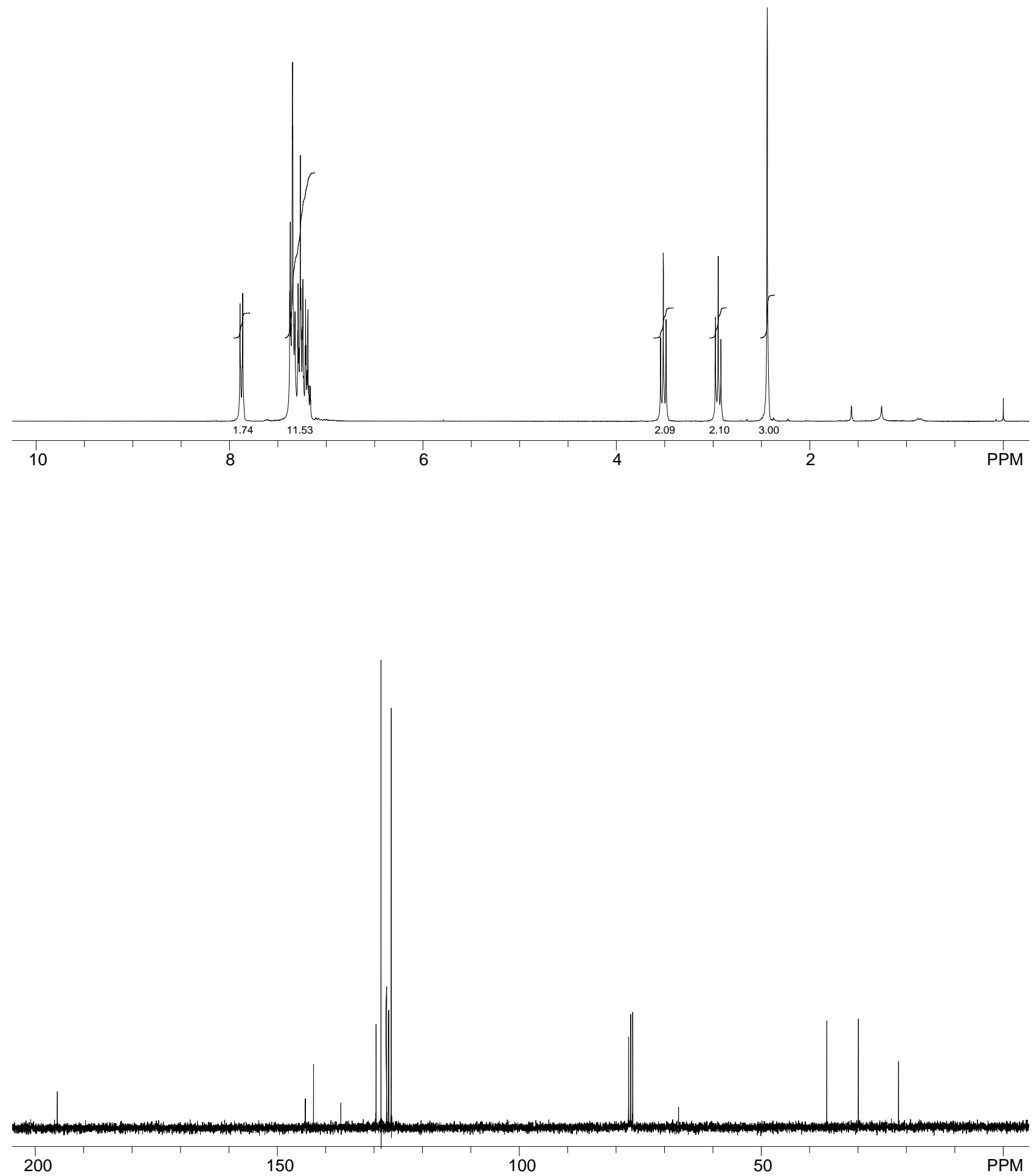

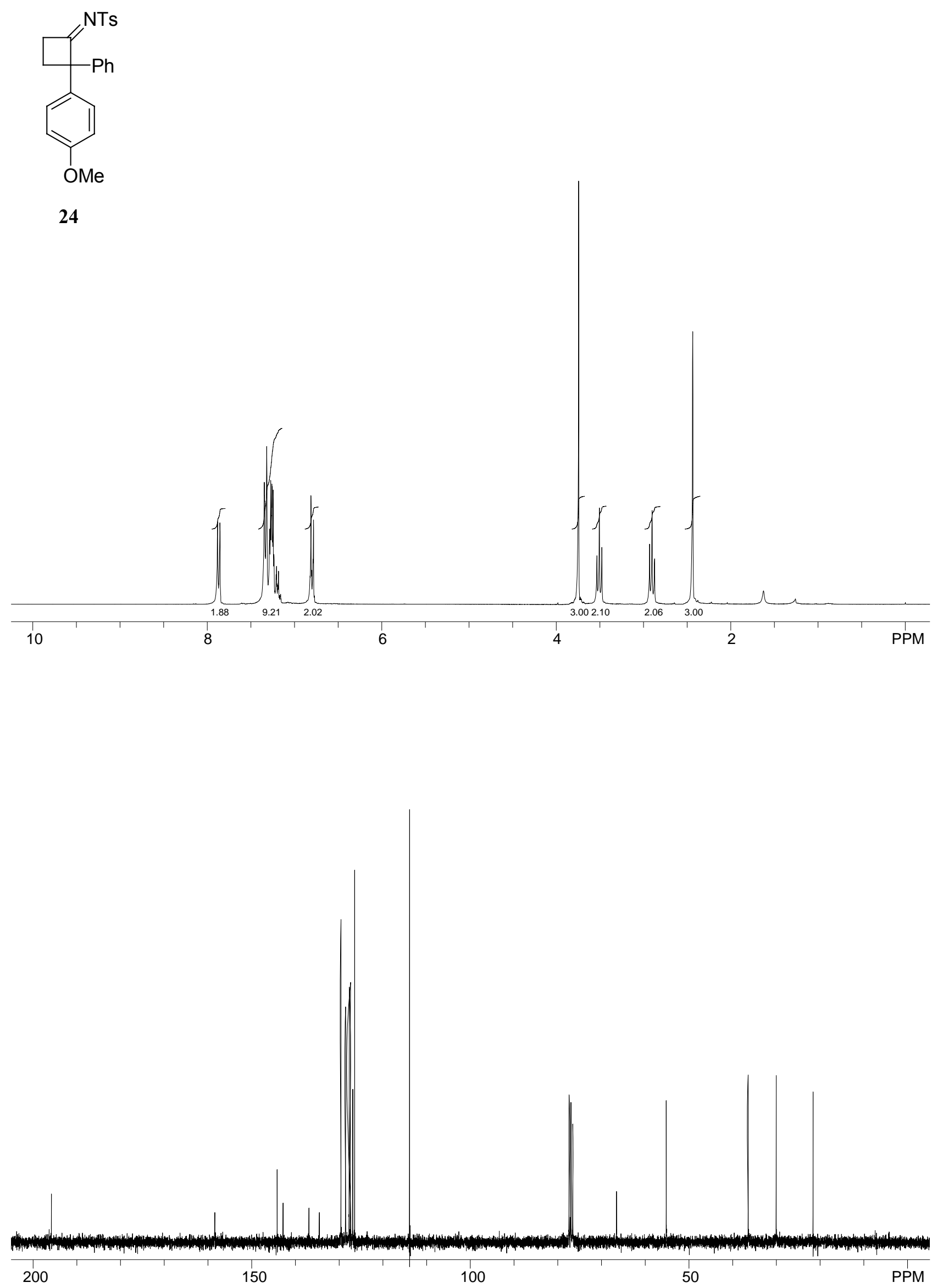


\section{Crystal Structure of Compound E-6}

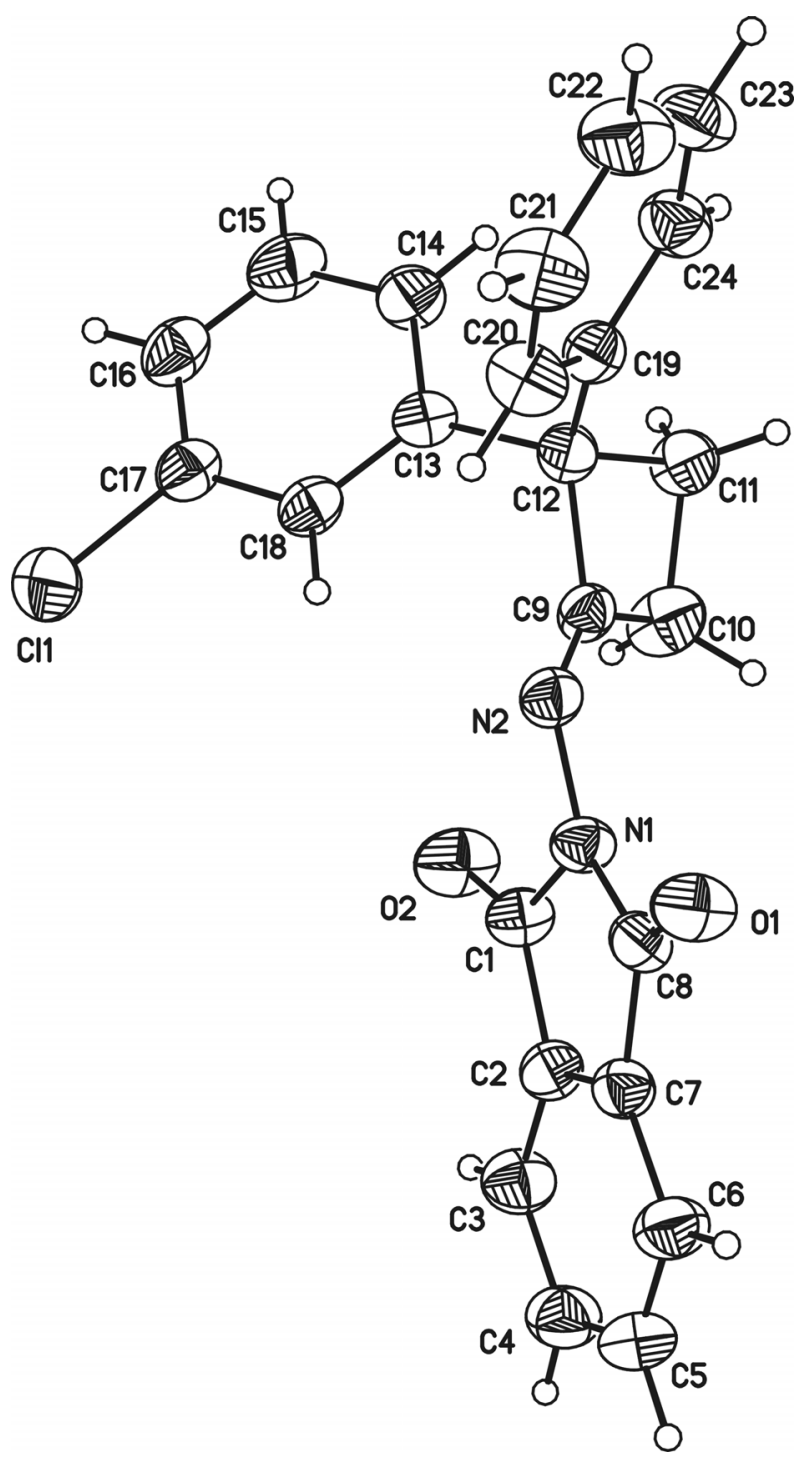

CCDC 619410 contains the supplementary crystallographic data for this paper. These data can be obtained free of charge via www.ccdc.cam.ac.uk/data_request/cif, by emailing data_request@ccdc.cam.ac.uk, or by contacting The Cambridge Crystallographic Data Centre, 12, Union Road, Cambridge CB2 1EZ, UK; fax: +44 1223336033. 